\title{
A Matter of Perspective
}

\section{Choosing for Others Differs from Choosing for Yourself in Making Treatment Decisions}

\author{
Brian J. Zikmund-Fisher, PhD, ${ }^{1,2,3}$ Brianna Sarr, BS, ${ }^{2,3}$ Angela Fagerlin, $P h D,{ }^{1,2,3}$ \\ Peter A. Ubel, MD ${ }^{1,2,3,4}$ \\ 'VA Health Services Research \& Development Center for Practice Management and Outcomes Research, VA Ann Arbor Healthcare \\ System, Ann Arbor, MI, USA; ${ }^{2}$ Center for Behavioral and Decision Sciences in Medicine, Ann Arbor, MI, USA; ${ }^{3}$ Division of General Internal \\ Medicine, University of Michigan, Ann Arbor, MI, USA; 'Department of Psychology, University of Michigan, Ann Arbor, MI, USA.
}

\begin{abstract}
BACKGROUND: Many people display omission bias in medical decision making, accepting the risk of passive nonintervention rather than actively choosing interventions (such as vaccinations) that result in lower levels of risk.
\end{abstract}

OBJECTIVE: Testing whether people's preferences for active interventions would increase when deciding for others versus for themselves.

RESEARCH DESIGN: Survey participants imagined themselves in 1 of 4 roles: patient, physician treating a single patient, medical director creating treatment guidelines, or parent deciding for a child. All read 2 short scenarios about vaccinations for a deadly flu and treatments for a slow-growing cancer.

PARTICIPANTS: Two thousand three hundred and ninety-nine people drawn from a demographically stratified internet sample.

MEASURES: Chosen or recommended treatments. We also measured participants' emotional response to our task.

RESULTS: Preferences for risk-reducing active treatments were significantly stronger for participants imagining themselves as medical professionals than for those imagining themselves as patients (vaccination: 73\% [physician] \& 63\% [medical director] vs $48 \%$ [patient], $P$ s <.001; chemotherapy: $68 \% \& 68 \%$ vs $60 \%, P \mathrm{~s}<.012$ ). Similar results were observed for the parental role (vaccination: $57 \%$ vs $48 \%$, $P=.003$; chemotherapy: $72 \%$ vs $60 \%, P<.001)$. Reported emotional reactions were stronger in the responsible medical professional and parental roles yet were also independently associated with treatment choice, with higher scores associated with reduced omission tendencies $(\mathrm{OR}=1.15$ for both regressions, $P \mathrm{~s}<.01)$.

CONCLUSIONS: Treatment preferences may be substantially influenced by a decision-making role. As certain roles appear to reinforce "big picture" thinking about difficult risk tradeoffs, physicians and patients should consider re-framing treatment decisions to gain new, and hopefully beneficial, perspectives.

KEY WORDS: decision making; risk communication; omission bias. DOI: $10.1111 /$ j.1525-1497.2006.00410.x J GEN INTERN MED 2006; 21:618-622.

$\mathrm{C}$ onsider the medical decision problem in Box 1. A deadly flu threatens your area, killing $10 \%$ of the population, and you must decide whether to get vaccinated. Although the vaccine protects you from the flu epidemic, it also causes a significant $(5 \%)$ mortality risk of its own. Taking the vaccine is the survival-maximizing choice. Doing so, however, may increase your sense of responsibility for any harm that may befall you.

The authors have no conflicts of interest to declare.

This work was presented at the annual meeting of the Society for Judgment and Decision Making, November 13, 2005.

Address correspondence and requests for reprints to Dr. ZikmundFisher, Center for Behavioral and Decision Sciences in Medicine, 300 North Ingalls Building, Rm. 7C27, Ann Arbor, MI 48109-0429 (e-mail: bzikmund@med.umich.edu).
Researchers have labeled this type of situation as an omission bias problem and have consistently shown that many people are willing to face higher risks of death to avoid having "caused" harm. ${ }^{1,2}$ In mail surveys of parents, those who demonstrated omission tendencies in hypothetical scenarios were less likely to report having their child vaccinated for pertussis and more likely to believe that vaccinating their child was more dangerous than not vaccinating, despite having received information to the contrary. ${ }^{3,4}$ Such omission tendencies primarily result from people drawing a sharp distinction between direct and indirect causation: People think about harms caused by direct actions much more than harms caused only indirectly. ${ }^{5}$

Susceptibility to omission "biases," however, may differ by what role people play in the decision-making process. Social roles and authority relationships affect how much people differentiate between harmful acts and harmful omissions. ${ }^{6}$ Specifically, if the actor holds a position with a high degree of responsibility, people's evaluations of a situation depend more on the outcome of the decision than on who caused any harms.

Why would role and authority matter? One possibility is that, when acting in roles with authority or social responsibility, people experience different emotional responses than when deciding for themselves. In particular, role may influence visceral reactions such as anxiety or worry that are linked to people's intuitive understandings of risk and underlie certain self-other differences in decision making. ${ }^{7}$ While causing harm is emotionally disturbing in every case, people might react differently to the prospect of hurting themselves versus causing harm to others as part of their duties or responsibilities. Alternately, responsibility may make people analyze decisions differently, focusing on certain components of the decision more than others. ${ }^{8,9}$

Role and perspective could have a significant impact on medical treatment decisions that compare active treatment approaches with more passive approaches. Such decisions commonly occur with life-threatening illnesses, because intensive, life-saving interventions often have their own inherent dangers. Despite the recent trend toward shared decision making, some patients still cede decision authority to their physicians and health care providers. ${ }^{10}$ But, do medical professionals make the choices patients would want them to? Ethically, the role of the physician is to help patients make the "best" decision, ${ }^{11}$ but patients may use different decisionmaking processes than physicians. Physicians' professional role and social distance might focus them more on the potential outcomes, whereas patients might focus more on how the

Manuscript received August 2, 2005

Initial editorial decision October 4, 2005

Final acceptance January 19, 2006 
outcomes are achieved (i.e., the story of their illness). ${ }^{12}$ Adopting a policy-making perspective could reinforce this difference. For example, hospital medical directors might have an even greater tendency toward objectivity than physicians, because their decisions affect more people and because they must act in the best interests of the majority even if doing so might bring harm to a single person. ${ }^{13,14}$

Another important perspective is the parental role. Parents' immunization decisions are strongly influenced by omission bias factors, such as anticipated responsibility and potential regret. ${ }^{15,16}$ While parents certainly have a sense of responsibility about their children, there is also a close emotional connection involved. Both factors could influence treatment decision making.

In this study, we examined the potential impact of professional and parental roles on omission tendencies in medical treatment decisions. To identify how perspective and role may affect decisions independent of the knowledge and experience associated with becoming a medical professional, we used a randomized experimental design to compare differently worded versions of 2 medical decision scenarios. To further elucidate why different roles might lead to different treatment decisions, we also assessed the degree to which taking on these roles evoked emotional responses and whether those responses affected treatment choices.

\section{METHODS \\ Overview of Study Design}

As part of a larger internet-administered survey on medical decision making, participants read descriptions of 2 medical decisions: (1) vaccination for a deadly flu virus and (2) treatment of a slow-growing cancer. We randomized participants to consider these decisions while imagining themselves in 1 of 4 possible roles and then assessed their treatment preferences. This design received Institutional Review Board exempt status approval.

\section{Participants}

Study participants were drawn from a panel of internet users, administered by Survey Sampling International (SSI), who all voluntarily agreed to receive invitations to fill out questionnaires. To ensure demographic diversity (but not representativeness), email invitations were sent to a stratified random sample of panel members weighted to offset expected differences in response rates (especially for African Americans and Hispanic Americans), with the goal of approximating the U.S. census on gender, education level, and income in the final subject pool. We also drew 3 distinct age samples to enable greater power for age-based analyses: one-third aged 21 to 40, one-third aged 41 to 64 , and one-third aged 65 and older. Upon completion, participants were entered into a drawing administered by SSI for cash prizes totaling $\$ 10,000$.

\section{Intervention}

The main experimental manipulation involved asking respondents to imagine themselves in 1 of 4 decision-making roles. Some participants were told they were at risk and were deciding their own course of treatment ("self" role). A second group imagined being physicians recommending a treatment

\section{Box 1: Flu Scenario.}

(Page 1)
Imagine that there will be a deadly flu going around your area next
winter. Your doctor says that you have a 10\% chance (10 out of 100)
of dying from this flu.
Take a moment to vividly imagine what it would be like to be in this
situation.
(Page 2)
A new flu vaccine has been developed and tested. If taken, the
vaccine prevents you from catching the deadly flu. However, there is
1 serious risk involved with taking this vaccine. The vaccine is made
from a somewhat weaker type of flu virus, and there is a $5 \%$ out of 5 ou
100) risk of the vaccine causing you to die from the weaker type of
flu.
Imagine that this vaccine is completely covered by health insurance.
(Page 3)
Remember, you are imagining that you could get a type of deadly
flu. Please give your best answer to the following questions.
If you had to decide now, which would you choose?
$\bullet$ I would not take the vaccine and accept the $10 \%$ chance of
dying from this flu.
- I would take the vaccine and accept the $5 \%$ chance of dying
from the weaker flu in the vaccine.

approach to a single patient of unspecified age and gender ("physician" role). A third group imagined acting as a hospital medical director setting treatment guidelines for all patients ("medical director" role). The final group imagined being parents of an at-risk minor child and making treatment decisions for their son or daughter ("parent" role). Participants were randomly assigned among role conditions.

Regardless of role, respondents read both the deadly flu vaccination scenario described above (Box 1) and a similarly structured slow-growing cancer scenario (Appendix A, online) in random order. Each scenario included an active treatment (taking the vaccine or undergoing chemotherapy) and a passive treatment approach (no vaccine or watchful waiting). In both cases, choosing active treatment reduced overall mortality by $5 \%$ versus the passive alternative. However, the mortality risk associated with active treatment was caused by the vaccine or chemotherapy, whereas the larger mortality risk associated with the other treatment choice occurred by chance.

Between scenarios, respondents also completed a brief measure, adapted from the PANAS, ${ }^{17}$ of the emotions evoked by their task. Participants were shown 10 emotion words (anxious, distressed, guilty, interested, conflicted, determined, uncomfortable, concerned, worried, and responsible) and rated how much reading the prior scenario made them feel each emotion on a 1 to 5 scale ( $1=$ "very slightly or not at all," $5=$ "extremely"). While we considered whether each scenario evoked certain emotions more than others, our primary measure was the average emotional rating, which we took to represent each respondent's overall level of emotional activation.

\section{Outcome Measures}

Our primary outcome measure was the treatment either chosen or recommended by each respondent for the 2 scenarios. 


\section{Hypotheses}

We predicted that imagining oneself as a health care professional (i.e., physician or medical director) would evoke a sense of duty to act in the "best interests" of patients and increase attention to harms of omission, leading to greater selection of active treatment versus participants in the self-role.

Past research suggests that people feel stronger emotions about risks for oneself versus risks for others. ${ }^{7}$ Omission contexts trade off 2 risks, however: higher (passive) mortality risk versus the risk of causing harm, both of which could evoke emotional reactions. Our a priori belief was that the latter risk would be less emotionally salient in health professional roles versus the self condition, reducing overall emotional activation and further reducing omission tendencies. We similarly anticipated that participants reporting weaker emotional reactions to our task would choose active treatment options more often. As older adults pay more attention to affect and are more influenced by affective cues (e.g., emotional appeals) than younger adults, ${ }^{18-21}$ the same logic suggests that younger subjects would be more likely to choose active treatment than our oldest participants (age 65 and older).

The parental role is more complex. While parental responsibility should reinforce the need to maximize the chance of the child surviving, the prospect of causing harm to one's own child might evoke particularly strong emotions, potentially offsetting this effect. As a result, we had no a priori hypotheses regarding behavior in the parent role condition. We did expect greater emotional reactions from "parent" respondents compared with the medical professional roles.

\section{Statistical Analysis}

We utilized $\chi^{2}$ tests of proportions to test whether treatment selections differed across role conditions and whether any respondent demographic characteristics were associated with treatment choice. We then used logistic regression to perform multivariate analyses including role condition, demographic variables, and reported emotion as potential explanatory variables. All analyses were performed using STATA 8.

\section{RESULTS}

A total of 30,375 people received email invitations, and 2,917 (a 9.6\% response rate) clicked the embedded link to see the survey cover page. While some dropout occurred as people read further, 2,399 participants (82.2\%) provided useable data.
Sample mean age was 51 (range 21 to 89), 47\% were male, and, of the 2,124 who reported racial and ethnic background information, $82 \%$ described themselves as Caucasian, 13\% Hispanic (any race), 10\% African American, 2\% Asian American, and 5\% multi-ethnic or other race. We observed a wide range of educational achievement, with $37 \%$ having completed a Bachelor's or higher degree but also $17 \%$ with only a high school education or less. Compared with nonrespondents (using information from SSI), participants were slightly older (mean age 51 vs 46 ) and more educated (37\% with Bachelor's or higher degree vs $32 \%$ ), due to oversampling of younger adults needed to offset lower response rates. Most importantly for testing our primary hypothesis of role effects, there were no significant variations in sample characteristics across the role conditions.

\section{Role and Decision Making}

Table 1 reports the percentage of survey participants who chose the active treatment option by both decision-making role and scenario type. In a clear demonstration of preferences for inaction, a significant minority of participants declined the survival-maximizing active treatment in all conditions, including the 2 medical professional roles. However, the first 3 columns show that, as predicted, the percentage of people choosing active treatments increased significantly for subjects imagining themselves in a professional role, compared with the self role. The effect size was noticeably larger with the flu vaccine scenario than for the slow-growing cancer scenario. In the flu scenario, "medical directors" were more likely to support vaccination than even "physicians" $\left(\chi^{2}(1)=13.29, P<.001\right)$. No such difference was observed in the cancer scenario.

The fourth column of Table 1 reports the results from the parental role conditions. Here we observed a significant difference between the 2 scenarios. In the slow-growing cancer scenario, people who imagined making a treatment decision for their own child were more likely than all others to choose active chemotherapy over watchful waiting. By contrast, in the flu scenario, "parent" respondents were more likely to choose active treatment (i.e., the vaccine) than respondents in the selfrole but less likely to do so than respondents in the medical professional roles.

Within each role condition, treatment choices were similar for the 2 youngest age cohorts (ages 21 to 40 and 41 to 64) in both scenarios. However, respondents 65 years of age and older were significantly less likely to undergo chemotherapy in the slow-growing cancer scenario $(M=58.9 \%$ vs $70.6 \%$, $\chi^{2}(1)=28.49, P<.001$, consistent with our age hypothesis)

Table 1. Effect of Imagined Decision-Making Role on Willingness to Choose Active Treatment

\begin{tabular}{|c|c|c|c|c|}
\hline & \multicolumn{4}{|c|}{ Decision-Making Role } \\
\hline & Self $(n=629)$ & Physician $(n=554)$ & Medical Director $(n=550)$ & Parent $(n=586)$ \\
\hline Deciding for & Oneself & 1 Patient & All Patients & Own Child \\
\hline \multicolumn{5}{|l|}{ Flu scenario } \\
\hline$\%$ taking the vaccine & 48 & 63 & 73 & 57 \\
\hline$\chi^{2}$ test $(1 d f)$ vs self role ${ }^{*}$ & - & $P<.001$ & $P<.001$ & $P=.003$ \\
\hline \multicolumn{5}{|l|}{ Slow growing cancer scenario } \\
\hline \% choosing chemotherapy & 60 & 68 & 68 & 72 \\
\hline$\chi^{2}$ test ( $1 d f$ ) vs self role & - & $P=.008$ & $P=.012$ & $P<.001$ \\
\hline
\end{tabular}

*In the flu scenario, all pairwise comparisons between the physician, medical director, and parent roles are also significant at $\mathrm{P}<.05$ or better. 
Table 2. Logistic Regression Analyses of the Selection of Active Treatment Options

\begin{tabular}{|c|c|c|c|c|c|c|}
\hline \multirow{2}{*}{$\begin{array}{l}\text { Dependent Variable: Choice of the active treatment option } \\
\text { Variable }\end{array}$} & \multicolumn{3}{|c|}{ Flu Scenario } & \multicolumn{3}{|c|}{ Slow Growing Cancer Scenario } \\
\hline & Odds Ratio & $z$ & P-Value & Odds Ratio & $z$ & P-Value \\
\hline Physician role (vs self) & 1.71 & 4.25 & $<.001$ & 1.39 & 2.56 & .011 \\
\hline Medical director role (vs self) & 2.92 & 8.10 & $<.001$ & 1.38 & 2.49 & .013 \\
\hline Parent role (vs self) & 1.38 & 2.56 & .010 & 1.65 & 3.71 & $<.001$ \\
\hline Average emotion rating & 1.15 & 2.70 & .007 & 1.15 & 2.56 & .010 \\
\hline Age 41 to 64 (vs $\leq 40)$ & 0.87 & -1.22 & .212 & 0.74 & -2.54 & .011 \\
\hline Age $65+(\mathrm{vs} \leq 40)$ & 1.43 & 3.09 & .002 & 0.50 & -5.84 & $<.001$ \\
\hline Male gender & 1.24 & 2.30 & .022 & 0.98 & -0.22 & .823 \\
\hline Overall $\chi^{2}(7 d f)$ & & 105.51 & & & 62.09 & \\
\hline
\end{tabular}

but significantly more likely to take the vaccine in the deadly flu scenario $\left(M=66.4 \%\right.$ vs $56.3 \%, \chi^{2}(1)=19.39, P<.001$, contrary to expectations but consistent with the strong provaccination messages targeted at older adults). This pattern of behavior was not role specific; similar significant age effects were observed in all 4 role conditions, albeit at different baseline likelihoods of choosing active treatment.

\section{Affect and Decision Making}

Our aggregate measure of emotional activation was significantly related to participants' imagined role, but not in accord with our hypotheses. Scores were highest for respondents in the parent role $(M=2.93)$, next highest for the physician and medical director roles $(M=2.55$ and 2.56 , respectively), and lowest for the self role $(M=2.31)$. All pairwise comparisons (except physician vs medical director) were highly significant at $P<.001$. Female participants reported higher average scores than male subjects $(M=2.69$ vs $2.46, t=5.75, P<.001)$, but there was no significant interaction with respondent age. Our decision to rely on an aggregate measure was supported by the very similar response patterns for each of the 10 emotion words. The findings are qualitatively similar even if only a subset of the emotion responses are used.

We used a multivariate logistic regression framework to test whether emotional activation would predict treatment choice independent of its association with role. The results, shown in Table 2, confirmed the main effects of role and age discussed above and identified a gender effect in the flu scenario. The emotion variable did have a small but significant independent effect, with greater reported emotion levels resulting in greater selection of active treatment in both scenarios. Role remained a significant predictor even when emotion was added to the regressions, suggesting that role acts on the decision-making process in broader ways than just increasing emotional activation.

\section{DISCUSSION}

Our results demonstrate that medical treatment decisions can vary, often significantly, simply by changing the decision maker's perspective. Compared with respondents imagining themselves as patients, participants imagining themselves as medical professionals making treatment recommendations to patients (either individually or collectively) were significantly more likely to choose the survival-maximizing actions of undergoing chemotherapy and flu vaccination. This shift occurred even though our research participants had neither the formal training nor the professional experience of true physicians. Their propensity to choose active treatment changed simply as a result of thinking about the problem from an alternate perspective.

Our findings are consistent with previous research, which suggests that, while people making decisions for themselves consider many different dimensions, people giving advice to others focus on a single dimension. ${ }^{8,22}$ After all, advice givers may be asked to justify their decisions to others ${ }^{9}$ or feel a need to justify them to themselves, ${ }^{23}$ and maximizing survival chances is easy to defend. Our results also support previous findings that people accept medical interventions more often when choosing on behalf of another than when choosing for themselves. $^{24}$

Our aggregate measure of respondents' emotions was significantly related to treatment choices, although not as expected. Greater emotional activation mediated the role effect and was independently correlated with choosing active treatment. Clearly, we measured something important, although "emotion" may not be the best label for this construct. Life and death medical decisions are obviously emotional, yet the low emotion scores of the "patient" respondents suggest that we failed to pick up on these affective responses. A better label for what we measured might be engagement. Imagining being responsible for a sick child or giving advice to patients may have been more engaging than imagining (counterfactually) facing an imminent risk of death. Greater engagement would have induced people to focus on outcomes (i.e., survival) and thus choose active treatment. The engagement concept is also consistent with other unpublished research we have conducted showing that imagining a high responsibility role reduces protest responses in utility elicitation exercises.

Although this research did not involve actual treatment decisions by patients or health care providers, it suggests that physician advice is still an important role function. ${ }^{25}$ Physician recommendations have a powerful impact on patient decisions, ${ }^{26}$ and the advent of patient involvement and shared decision making in medicine has led some physicians to back away from expressing their own preferences, believing instead that their role should focus primarily on providing information rather than guiding choices. ${ }^{27,28}$ Our results suggest that such an approach may be problematic. Some patients may fear having "caused" their own harm if an active treatment turns out poorly yet at the same time recognize the advantages of survival-maximizing active treatments when choosing for another. These patients may benefit from receiving treatment recommendations from their physician (or family members). In particular, providers may want to consider asking patients to 
"reframe" the decision they face and consider it as if they were choosing for someone else. This shift in perspective may help some patients better understand the tradeoffs they face.

Our research has several limitations. First, our survey used an internet sample, which, although demographically diverse, may be nonrepresentative in unmeasured ways. Our goal, however, was not to achieve a representative sampling frame but instead to compare treatment choices between experimental groups. Our randomized design supports internal validity by distributing sample peculiarities evenly across role conditions, thus controlling for any response biases possibly indicated by our low response rate. Second, our use of Internet subjects and hypothetical scenarios may have reduced participants' motivation to take the task seriously, inflating tendencies to choose passive treatment approaches. It seems likely that people might be more survival focused when actually facing life-threatening illnesses. Third, some subjects may have misunderstood the risk information presented, increasing error. Still, our experimental results should remain valid unless these factors had a greater impact on decision making in the self role condition than on decisions in the other 3 role conditions.

Medical decisions should be fact driven. Given a particular decision problem and a consistent set of facts, we expect physicians and patients to see the situation similarly and thus make similar decisions. Our results show that what seems reasonable for yourself may seem less appropriate when giving advice or acting on behalf of another, even though your personal values are the same in both cases. Although decision role matters, we do not assert a single "correct" perspective. We do suggest that medical professionals should reconsider the role they play in facilitating patient decision making. What we choose for ourselves is not always what we would choose for another, and reflecting on this fact may help both patients and physicians to improve their decisions.

Financial support for this study was provided by grants from the National Institutes for Health (R01 CA87595 and P50 CA 101451). Dr. Fagerlin is supported by an MREP early career award from the Department of Veterans Affairs. The funding agreements ensured the authors\&apos: independence in designing the study, interpreting the data, and publishing the report.

1. Ritov I, Baron J. Reluctance to vaccinate: omission bias and ambiguity. J Behav Decis Making. 1990;3:263-77.

2. Spranca M, Minsk E, Baron J. Omission and commission in judgment and choice. J Exp Soc Psychol. 1991;27:76-105.

3. Asch DA, Baron J, Hershey JC, et al. Omission bias and pertussis vaccination. Med Decis Making. 1994;14:118-23.

4. Meszaros JR, Asch DA, Baron J, Hershey JC, Kunreuther H, Schwartz-Buzaglo J. Cognitive processes and the decisions of some parents to forego pertussis vaccination for their children. J Clin Epidemiol. 1996;49:697-703.

5. Baron J, Ritov I. Omission bias, individual differences, and normality. Organ Behav Hum Decis Process. 2004;94:74-85.

6. Haidt J, Baron J. Social roles and the moral judgement of acts and omissions. Eur J Soc Psychol. 1996;26:201-18.

7. Loewenstein GF, Weber EU, Hsee CK, Welch N. Risk as feelings. Psychol Bull. 2001;127:267-86.

8. Kray L, Gonzalez R. Differential weighting in choice versus advice: I'll do this, you do that. J Behav Decis Making. 1999;12:207-17.

9. Jonas E, Schulz-Hardt S, Frey D. Giving advice or making decisions in someone else's place: the influence of impression, defense, and accuracy motivation on the search for new information. Pers Soc Psychol Bull. 2005;31:977-90.

10. Demoratz MJ. Advance directives: getting patients to complete them before they need them. Case Manager. 2005;16:61-3.

11. Lantos J. Informed consent. The whole truth for patients?. Cancer. 1993;72 (suppl 9):2811-5.

12. Kurzon N. A poker player's guide to beating cancer. Newsweek 2004, January 19, 2004:12.

13. Asch DA, Hershey JC. Why some health policies don't make sense at the bedside. Ann Intern Med. 1995;122:846-50.

14. Chant AD. Practising doctors should not manage. Lancet. 1984;1: 1398.

15. Wroe AL, Turner N, Salkovskis PM. Understanding and predicting parental decisions about early childhood immunizations. Health Psychol. 2004;23:33-41.

16. Wroe AL, Bhan A, Salkovskis P, Bedford H. Feeling bad about immunising our children. Vaccine. 2005;23:1428-33.

17. Watson D, Clark LA, Tellegen A. Development and validation of brief measures of positive and negative affect: the PANAS scales. J Pers Soc Psychol. 1988;54:1063-70.

18. Carstensen LL, Isaacowitz DM, Charles CT. Taking time seriously: a theory of socioemotional selectivity. Am Psychol. 1999;54:165-81.

19. Charles S, Mather M, Carstensen LL. Aging and emotional memory: the forgettable nature of negative images for older adults. J Exp Psychol Gen. 2003; 132:310-24.

20. Peters E, Finucane ML, MacGregor DG, Slovic P. The bearable lightness of aging: judgment and decision processes in older adults. In: Stern PC, Carstensen LL, eds. The Aging Mind: Opportunities in Cognitive Research. Washington, DC: National Research Council, National Academy Press; 2000: 144-65.

21. Williams $\mathbf{P}$, Drolet A. Age-related differences in responses to emotional advertisements. J Consum Res. 2005;32:343-54.

22. Kray LJ. Contingent weighting in self-other decision making. Organ Behav Hum Decis Process. 2000;83:82-106.

23. Connolly T, Zeelenberg M. Regret in decision making. Psychol Sci. 2004; 11:212-6.

24. Raymark P. Accepting or rejecting medical treatment: a comparison of decisions made for self versus those made for a significant other. J Appl Soc Psychol. 2000;30:2409-36.

25. Ubel PA. "What should I do, doc?": some psychologic benefits of physician recommendations. Arch Intern Med. 2002;162:977-80.

26. Gurmankin AD, Baron J, Hershey JC, Ubel PA. The role of physicians recommendations in medical treatment decisions. Med Decis Making. 2002;22:262-71.

27. McNutt RA. Shared medical decision making: problems, process, progress. JAMA. 2004;292:2516-8.

28. Schneider CE. The Practice of Autonomy: Patients, Doctors, and Medical Decisions. Oxford: Oxford University Press; 1998.

\section{Supplementary Material}

The following supplementary material is available for this article online at www.blackwell-synergy.com

Appendix A. A Matter of Perspective: Choosing for Others Differs from Choosing for Yourself in Making Treatment Decisions. 